\title{
Can we identify individuals with an $A L P L$ variant in adults with persistent hypophosphatasaemia?
}

\author{
C. Tornero ${ }^{1 *}$, V. Navarro-Compán ${ }^{1}$, J. A. Tenorio ${ }^{2,3}$, S. García-Carazo ${ }^{1}$, A. Buño ${ }^{4}$, I. Monjo ${ }^{1}$, C. Plasencia-Rodriguez ${ }^{1}$, \\ J. M. Iturzaeta ${ }^{4}$, P. Lapunzina ${ }^{2,3}$, K. E. Heath ${ }^{2,3,5}$, A. Balsa ${ }^{1}$ and P. Aguado ${ }^{1}$
}

\begin{abstract}
Background: Hypophosphatasia (HPP) is an inborn error of metabolism characterized by low levels of serum alkaline phosphatase (ALP). Scarce evidence exists about features that should signal the potential association between hypophosphatasaemia and HPP in adults. The aim of this study is to estimate the prevalence of ALPL variants in subjects with persistent hypophosphatasaemia and determine the associated clinical and laboratory features. For this cross-sectional study, laboratory records of 386,353 subjects were screened by measurement of ALP activity. A total of $85(0.18 \%)$ subjects with persistent hypophosphatasaemia ( $\geq 2$ serum alkaline phosphataseALP-measurements $\leq 35 \mathrm{IU} / \mathrm{L}$ and none $>45 \mathrm{IU} / \mathrm{L}$ ) were included (secondary causes previously discarded). ALPL genetic testing and a systematized questionnaire to retrieve demographic, clinical and laboratory data were performed. Descriptive analysis and logistic regression models were employed to identify the clinical and laboratory characteristics associated with ALPL variants.
\end{abstract}

Results: Forty subjects (47\%) had a variant(s) in ALPL. With regard to clinical characteristics, the presence of an ALPL variant was significantly associated only with musculoskeletal pain (OR: 7.6; 95\% IC: 1.9-30.9). Nevertheless, a trend to present more dental abnormalities (OR: 3.6; 95\% IC: 0.9-13.4) was observed. Metatarsal stress fractures were also more frequent ( 4 vs $0 ; p<0.05$ ) in this group. Regarding laboratory features, median ALP levels were lower in subjects with ALPL variants ( 26 vs $29 \mathrm{IU} / \mathrm{L} ; p<0.005$ ). Interestingly, the threshold of ALP levels $<25 \mathrm{IU} / \mathrm{L}$ showed a specificity, positive predictive value and positive likelihood ratio of $97.8,94.4 \%$ and 19.8 to detect a positive ALPL test, respectively.

Conclusions: In subjects with persistent hypophosphatasaemia -secondary causes excluded- one out of two presented ALPL variants. Musculoskeletal pain and ALP levels $<25 \mathrm{IU} / L$ are associated with this variant(s). In this scenario, ALP levels $<25 \mathrm{IU} / \mathrm{L}$ seem to be very useful to identify individuals with the presence of an ALPL variant.

Keywords: Metabolic bone diseases, Hypophosphatasia, Hypophosphatasaemia, Alkaline phosphatase, ALPL

\section{Background}

Hypophosphatasia (HPP) is an inborn error of metabolism characterized by low levels of serum alkaline phosphatase (ALP) caused by loss-of-function variants. This results in variants in $A L P L$, located on chromosome 1p36.1-p34, which encodes the tissue-nonspecific alkaline phosphatase $[1,2]$. Impaired ALP activity can lead

\footnotetext{
* Correspondence: carolina.tornero@salud.madrid.org

${ }^{1}$ Department of Rheumatology, La Paz University Hospital, IdiPAZ, Paseo de la Castellana, 261, 28046 Madrid, Spain

Full list of author information is available at the end of the article
}

to the extracellular accumulation of ALP-specific substrates, such as inorganic pyrophosphate $(\mathrm{PPi})$, a potent inhibitor of bone and dental mineralization [3].

HPP is a rare disease with an estimated prevalence in Europe of $1 / 300,000$ in severe cases and of $1 / 6370$ in moderate cases [4]. HPP covers a wide spectrum of clinical manifestations whose severity inversely correlates with the age of onset $[5,6]$. Clinical features range from lethal phenotypes or rickets in early ages to musculoskeletal pain, chondrocalcinosis, calcific periarthritis or fractures in adults $[5,7]$. 
The genetic background of subjects may impact the clinical course of HPP: recessive inherited variants are commonly associated with more severe manifestations, while both autosomal dominant and recessive inheritance may occur in milder forms [8]. Hence, efforts have been made to identify $A L P L$ disease-causing variants and their pattern of inheritance. To date, more than 380 variants have been described [9]. In addition, a high phenotypic variability within members of the same family sharing the same disease causing variants has been observed $[8,10]$.

The main biochemical hallmark of HPP is hypophosphatasaemia; however, in clinical practice, low ALP levels are often overlooked and their causes are not usually investigated $[6,11]$, even when evaluating bone fragility. This lack of clinical attention often leads to erroneous diagnoses and prescription of contraindicated drugs in HPP, such as antiresorptive agents $[12,13]$.

Scarce evidence exists about features that should signal the potential association between hypophosphatasaemia and HPP in adults. Previous studies have shown a prolonged delay in the diagnosis of $\operatorname{HPP}[5,14,15]$ and access to the genetic testing is not always possible or rapid testing may be required.

In this context, the aims of this study were to estimate the prevalence of subjects with variants in $A L P L$ among those with persistent hypophosphatasaemia and to determine the clinical and laboratory features associated with the presence of $A L P L$ variants and their utility to identify a positive genetic test in the diagnostic work-up of HPP.

\section{Results}

\section{Study population}

Out of 386,353 subjects, screened by enzyme assay, 231, 805 adults had at least two ALP measurements, of whom 427 exhibited persistent hypophosphatasaemia (i.e. $\geq 2$ ALP values $\leq 35 \mathrm{IU} / \mathrm{L}$ and none $>45 \mathrm{IU} / \mathrm{L})$. Thirty-one subjects were excluded because of secondary causes of low ALP levels (detailed in Fig. 1) and 13 because they could not be contacted by telephone. A total of 383 individuals fulfilled the selection criteria and were contacted, of whom 274 declined to participate. Finally, 109 subjects were enrolled and 85 signed the informed consent for the genetic testing. Study overview and selection procedures are depicted in Fig. 1.

The prevalence of persistent hypophosphatasaemia in adults of our clinical setting was $0.18 \%$ (see Fig. 1). Genetic test was performed in 85 individuals: 39 (46\%) displayed pathogenic (P) or likely pathogenic (LP) variants in $A L P L$ (positive genetic test: +GT group), 45 (53\%) did not show any $\mathrm{P}$ or LP variants (negative genetic test: -GT group) and one subject had a variant of unknown significance (VUS). Segregation analysis in the family of this subject displaying a VUS could not be performed; consequently, this case was excluded from further statistical analysis.

Regarding the subjects who showed variants in $A L P L$, $75 \%$ (30/40) were heterozygous for a P variant, 20\% (8/ 40) for a LP variant, $2.5 \%(1 / 40)$ for a VUS, and one patient (2.5\%) diagnosed of infantile HPP harbored compound heterozygous pathogenic variants. This patient had a history of multiple recurrent fractures, while subjects displaying variants in heterozygosity exhibited a less severe phenotype. Nine subjects had variants previously associated with odonto-HPP. We identified four variants previously unreported in the literature: two pathogenic variants [c.388 389insG; p.(Val130Glyfs*6) and c.619G > C; p.(Gln207Glu)] and two likely pathogenic variants [c.317A > G; p.(Gln10 6Arg) and c.547G > A; p.(Asp183Asn)]. The substitutions p.(Gly112Arg), p.(Val128Met), p.(Glu291Lys) and p.(Thr11 5_Ala116dup) were each present in three subjects; the disease-causing variants p.(Arg152Cys), p.(Asp183Asn), p.(A sp378Gly), p.(Thr166Ile), p.(Gly491Arg) and p.(Glu191Lys) in two subjects and the rest were observed in single subjects. Thirty-four subjects (85\%) had missense disease-causing variants, three had duplications (7.5\%) and the rest had deletions, insertions or splice site mutations (one subject for each group, respectively). Most of the variants were located in exons 5,6 and 9, were predicted to have a damaging effect in silico pathogenicity prediction tools and were absent or existed at extremely low frequencies in gnomAD. Additional file 1: Table S1 summarizes the complete list of $A L P L$ variants and the clinical findings associated to each of them.

Demographic characteristics of study participants are shown in Table 1. Median (IQR) age was 45 (38-55) years in the overall population. Compared with the -GT group, the +GT group showed a lower percentage of females and had a higher body mass index.

\section{Clinical manifestations}

Clinical features of $+\mathrm{GT}$ and $-\mathrm{GT}$ groups are detailed in Table 2. In the univariable analysis, the presence of musculoskeletal pain, premature tooth loss, dental abnormalities, metatarsal stress fractures and history of orthopedic surgery were significantly higher in the +GT group. While metatarsal stress fractures were detected in four subjects in the + GT group, no subject reported this type of fracture in the -GT group.

Data from the knee and/or pelvis X-ray was available for 11 subjects: 5 in the +GT group and 6 in the -GT group. Among them, two subjects in the former had radiographic chondrocalcinosis signs (one of them with a previous diagnosis of pyrophosphate arthropathy), contrasting with a negative previous history in the -GT group. A higher VAS was observed in the $+\mathrm{GT}$ group [ $3(2-5)$ vs $1(0-5) ; p<0.05]$ although functional assessments, as measured by HAQ-DI were comparable in the two genetic groups. 


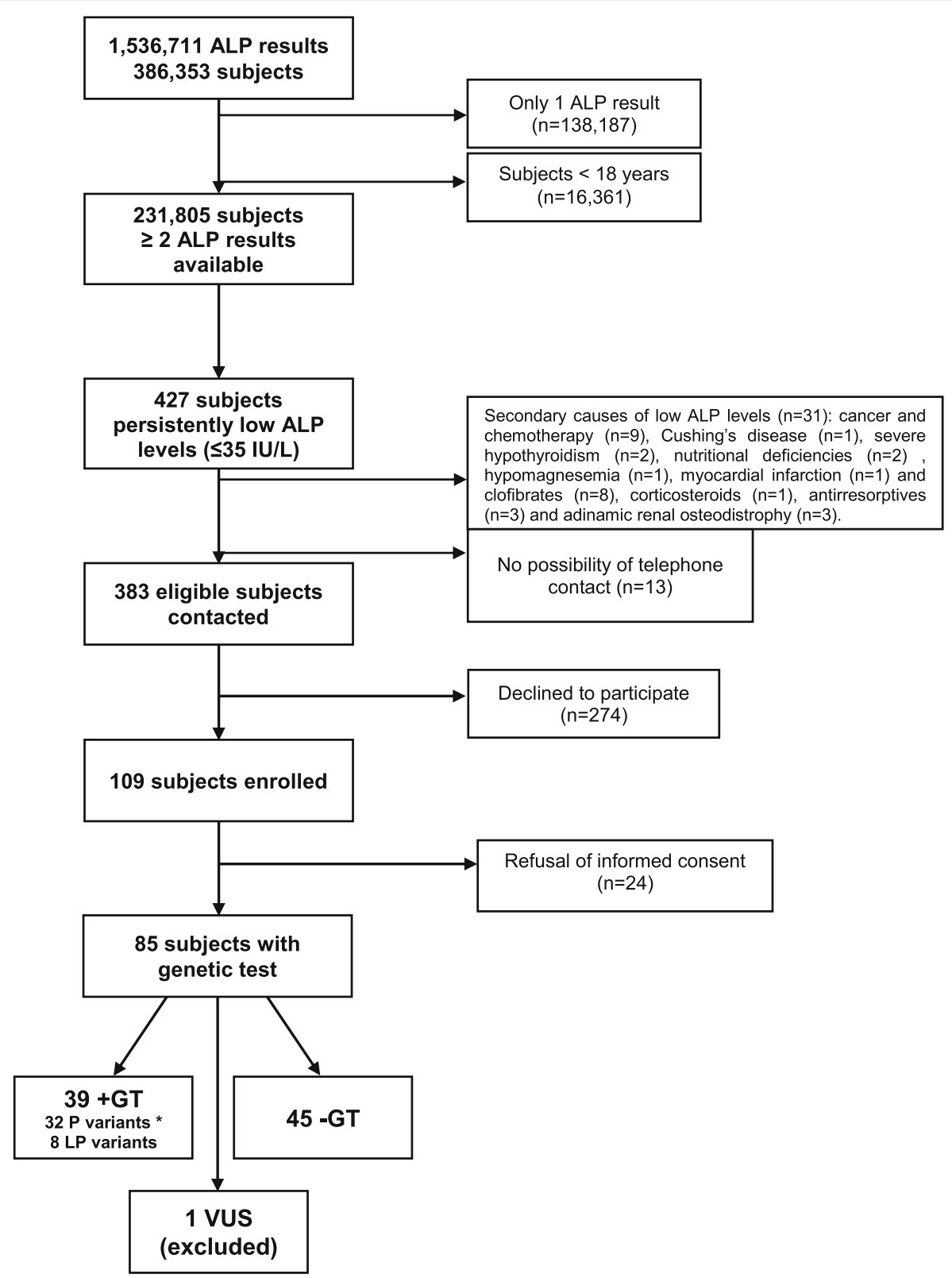

Fig. 1 Flowchart describing screening and selection process. ALP = alkaline phosphatase; $+G T$ and $-G T$ refer to subjects with either presence or absence of disease causing variants in $A L P L, P=$ pathogenic; $L P=$ likely pathogenic; VUS = Variant of unknown significance. ${ }^{*} T$ wo of the $P$ variants are present in one patient in compound heterozygosity

Furthermore, regression models adjusted for possible confounders (age, sex and body mass index) were employed to evaluate the association between clinical manifestations and ALPL disease causing variants. The results are shown in Table 3 . The only significant association with a positive genetic status was found for the presence of musculoskeletal pain (OR: 7.6; 95\% IC: 1.9-30.9). In addition, a trend was also detected for dental abnormalities (OR: 3.6; 95\% IC: 0.9-13.4). In this respect, nine subjects carried variants associated with odonto-HPP, of whom only five referred previous dental problems. Metatarsal stress fractures could not be analysed when adjusting for confounders because of convergence problems.

In the +GT group, a median 19-year delay between first signs or symptoms and diagnosis was observed, being the median (IQR) age at the onset of the symptoms of 40.3 years (13.6-50.5) and at the diagnosis of 49.8 (37.2-63.3) years. Median diagnostic delay was 22.7 (19.6-34.6) for those who experienced a pediatric onset $(n=9)$ and $11.9(7-21.2)$ for those who did not report symptoms before age $18(n=29)$. In terms of initial 
Table 1 Demographic characteristics of participants, stratified by ALPL genetic test

\begin{tabular}{|c|c|c|c|c|}
\hline & $+\mathrm{GT}^{*}(N=39)$ & $-\mathrm{GT}^{*}(N=45)$ & Total $(N=84)$ & $p$ value \\
\hline Median age (IQR), years & $49(37-63)$ & $44(38-48)$ & $45(38-55)$ & 0.092 \\
\hline Female sex, $\mathrm{n}(\%)$ & $23(59 \%)$ & $38(84.4 \%)$ & $61(72.6 \%)$ & $0.009^{* *}$ \\
\hline \multicolumn{5}{|l|}{ Race } \\
\hline Caucasian,n(\%) & 37 (94.8\%) & $45(100.0 \%)$ & $82(97.6 \%)$ & 0.3 \\
\hline Hispanoamerican, n(\%) & $1(2.6 \%)$ & 0 & $1(1.2 \%)$ & \\
\hline Black,n(\%) & $1(2.6 \%)$ & 0 & $1(1.2 \%)$ & \\
\hline Median $\mathrm{BMI} \mathrm{I}^{*}(\mathrm{IQR}), \mathrm{kg} / \mathrm{m}^{2}$ & $24.6(23.1-28.8)$ & $22.2(20.4-24.3)$ & $23.5(21.3-26.6)$ & $0.01^{* *}$ \\
\hline
\end{tabular}

*GT positive genetic test, $-G T$ negative genetic test, $B M I$ body mass index. ${ }^{*}$ Significant statistical differences between groups

HPP-associated symptoms, 8 subjects reported dental problems at a median age at onset of 14.51 (7.7-19.5); 18 , musculoskeletal symptoms at a median age at onset of 43 (14.8-51.8) and 2 of them, kidney complications.

\section{Laboratory findings}

The biochemical profile of study participants is presented in Table 4. ALP median (IQR) serum levels were significantly lower in the +GT group compared to those in the GT group [26 IU/L (22-29) vs 29 IU/L (27-32), $p<0.005]$. In contrast, median phosphate levels were significantly higher in the +GT group compared with the -GT group [4 $\mathrm{mg} / \mathrm{dL}(3.5-4.5)$ vs $3.4 \mathrm{mg} / \mathrm{dL}(3.1-4.1), p<0.05$, respectively). Eleven percent (5/44) of subjects, based on the available data, presented hyperphosphoremia $(>4.5 \mathrm{mg} / \mathrm{dL})$, most (4/44) in the +GT group. Median calcium levels were within the normal range, statistically comparable in both groups, and hypercalcemia was not observed in subjects using the available data. Levels of 24-h urinary calcium and phosphate excretion did not differ between groups.

The utility of identifying the presence of $A L P L$ variants based on different ALP cut-off levels (20, 25, 30 and 35 IU/ L) was assessed (Table 4). The threshold of $25 \mathrm{IU} / \mathrm{L}$ served as the best predictor of a positive $A L P L$ genetic test in the current study population. Of the 18 subjects with ALP levels below $25 \mathrm{IU} / \mathrm{L}, 17$ had a positive genetic test and only one a negative result. The values for sensitivity, specificity, positive and negative predictive value, and positive and negative likelihood ratio for ALP levels below $25 \mathrm{IU} / \mathrm{L}$ were 43.6, 97.8, 94.4, 66.7\%, 19.8 and 0.58, respectively (see Table 5).

Of the 65 subjects with ALP levels between 25 and 35 $\mathrm{IU} / \mathrm{L}$, we evaluated whether those clinical symptoms potentially related to HPP could improve the detection of a positive variant in our population. In the 36 subjects who presented musculoskeletal pain, 15 (41.7\%) displayed a positive variant and 21 (58.3\%), a negative genetic test.

Table 2 Clinical manifestations of participants stratified by $A L P L$ genetic testing

\begin{tabular}{|c|c|c|c|c|}
\hline Clinical feature, n (\%) & $+\mathrm{GT}^{*}(N=39)$ & $-\mathrm{GT}^{*}(N=45)$ & Total $(N=84)$ & $p$ value \\
\hline Musculoskeletal pain & $31(79.5 \%)$ & $21(46.7 \%)$ & $52(61.9 \%)$ & $0.002^{* *}$ \\
\hline \multicolumn{5}{|l|}{ Fractures } \\
\hline History of fractures & $17(43.6 \%)$ & $15(33.3 \%)$ & $32(38.1 \%)$ & 0.334 \\
\hline Multiple fractures & $2(5.0 \%)$ & $0(0.0 \%)$ & $2(2.4 \%)$ & 0.129 \\
\hline Peripheral fractures & $18(45.0 \%)$ & $13(28.9 \%)$ & $31(36.5 \%)$ & 0.124 \\
\hline Metatarsal fractures & $4(10.0 \%)$ & 0 & $4(4.7 \%)$ & $0.007^{* *}$ \\
\hline Family history of fractures & $6(15.8 \%)$ & $9(20.0 \%)$ & $15(18.1 \%)$ & 0.619 \\
\hline Orthopedic surgery & $7(18.4 \%)$ & $2(4.4 \%)$ & $9(10.8 \%)$ & $0.041^{* *}$ \\
\hline History of premature teeth loss & $6(15.4 \%)$ & $1(2.2 \%)$ & $7(8.3 \%)$ & $0.029^{* *}$ \\
\hline Dental abnormalities & $12(31.6 \%)$ & $6(13.3 \%)$ & $18(21.7 \%)$ & $0.045^{* *}$ \\
\hline Family history of dental problems & $11(35.5 \%)$ & $14(34.1 \%)$ & $25(34.7 \%)$ & 0.906 \\
\hline Muscle weakness & $6(15.4 \%)$ & $3(6.7 \%)$ & $9(10.7 \%)$ & 0.198 \\
\hline Calcific periarthritis & $4(10.3 \%)$ & $3(6.7 \%)$ & 7 (8.3\%) & 0.553 \\
\hline Chondrocalcinosis & $2(5,1 \%)$ & 0 & $2(2,4 \%)$ & 0.124 \\
\hline Median VAS* (IQR) & $3(2-5)$ & $1(0-5)$ & $2(0-5)$ & $0.038^{* *}$ \\
\hline Median HAQ-DI* (IQR) & $0(0-0.3)$ & $0(0-0.1)$ & $0(0-0.3)$ & 0.872 \\
\hline
\end{tabular}

*+GT positive genetic test, - GT negative genetic test, VAS Visual Analog Scale, HAQ-DI Health Assessment Questionnaire-Disability Index. **Significant statistical differences between groups 
Table 3 Results of the logistic regression model adjusted for possible confounders (age, sex and BMI) showing the association between the clinical features and genetic status

\begin{tabular}{llll}
\hline Clinical feature & Odds Ratio & $95 \%$ IC & $p$ value \\
\hline Musculoskeletal pain & 7.6 & $1.9-30.9$ & $0.005^{* *}$ \\
Peripheral fractures & 1.2 & $0.4-4$ & 0.769 \\
Family history of fractures & 0.4 & $0.06-2.2$ & 0.273 \\
Orthopedic surgery & 3.7 & $0.5-27.1$ & 0.199 \\
History of premature tooth loss & 1.7 & $0.1-21.9$ & 0.673 \\
Dental abnormalities & 3.6 & $0.9-13.4$ & 0.053 \\
Family history of dental problems & 0.7 & $0.2-2.3$ & 0.513 \\
Muscle weakness & 3.1 & $0.4-27.6$ & 0.309 \\
Calcific periarthritis & 1.5 & $0.2-9.9$ & 0.680 \\
\hline
\end{tabular}

**Significant statistical differences between groups

In addition, 12 subjects reported dental abnormalities (half having an $A L P L$ variant) and two individuals diagnosed with HPP experienced metatarsal fractures. The specificity and positive predictive value for musculoskeletal pain in this group of subjects were 52.3 and $41.7 \%$, respectively and 86.4 and $50 \%$ for dental abnormalities; thus, the discriminative power did not increase (Additional file 2: Table S2).

\section{Discussion}

Persistently low ALP levels can stem from different etiologies, including HPP. Characterization of the HPP spectrum in adults, which is generally characterized by milder symptoms than in pediatric-onset HPP, is crucial for the correct management and treatment of the disease. In addition, an accurate clinical and biochemical characterization can help distinguish between congenital HPP and secondary hypophosphatasaemia.

In this context, the results of our study are very relevant. Besides establishing the prevalence of an ALPL disease-causing variant in adult subjects with persistent hypophosphatasaemia, this is the first study utilizing a common biomarker to identify a positive genetic $A L P L$ test.

The prevalence of persistent hypophosphatasaemia in adults of our clinical setting was $0.18 \%$, which is in agreement with two studies, one conducted in a large rural multispecialty clinic population in the USA (prevalence $0.06 \%$ ) [11] and another in a French tertiary hospital $(0.13 \%)$ [6]. In our cohort, the subjects included were mainly Caucasians, aged around 50 years and predominantly female, which was congruent with other recent studies $[5,16,17]$. Furthermore, the estimated prevalence of $A L P L$ disease-causing variants in these subjects with persistent hypophosphatasaemia was $47 \%$. This means that one out of two subjects with persistent hypophosphatasaemia (secondary causes discarded) had HPP, which is in accordance with the study of Riancho-Zarrabeitia and coworkers [18]. More recently, the study of Mckiernan et al. [19] found a higher proportion of subjects with $A L P L$ disease-causing variants (84\%), most likely because a more stringent definition of hypophosphatasaemia was used. We identified four previously unreported variants based on the American College of Medical Genetics and Genomics (ACMG) classification criteria [20]. The majority of the variants were missense in nature and were located in exons five, six and nine, as has been commonly observed in public databases and previous reports $[9,18]$.

According to our results, approximately $50 \%$ of persistently low APL levels signify a different underlying etiology than HPP. Therefore, genetic confirmation by $A L P L$ genetic testing is required. Nevertheless, in clinical practice, access to such testing is not always available or rapid testing is required. Therefore, it would be very useful to identify the clinical and routine biochemical characteristics of the disease and its predictive value for a proper and early diagnosis, in order to avoid erroneous therapeutic decisions.

Based on the results of this study, neither single nor combined clinical manifestation are useful enough for

Table 4 Biochemical variables of study participants, stratified by genetic test results

\begin{tabular}{|c|c|c|c|c|}
\hline Biochemical variables, [Median (IQR)] & $+\mathrm{GT}^{*}(N=39)$ & $-\mathrm{GT}^{*}(N=45)$ & Total $(N=84)$ & $p$ value \\
\hline $\mathrm{ALP}^{*}, \mathrm{IU} / \mathrm{L}(\mathrm{N}=39,45,84)$ & $26(22-29)$ & $29(27-32)$ & $28(25-31)$ & $0.001^{* *}$ \\
\hline$<20 \mathrm{IU} / \mathrm{L}$ & $6(15 \%)$ & $0(0 \%)$ & $6(7.1 \%)$ & \\
\hline$<25 \mathrm{IU} / \mathrm{L}$ & $17(44 \%)$ & $1(2 \%)$ & $18(21 \%)$ & \\
\hline$<30 \mathrm{IU} / \mathrm{L}$ & $32(82 \%)$ & $25(56 \%)$ & $57(68 \%)$ & \\
\hline$<35 \mathrm{IU} / \mathrm{L}$ & $38(97 \%)$ & $43(96 \%)$ & $81(96 \%)$ & \\
\hline Calcium, mg/dL $(N=34,44,78)$ & $9.4(9.1-9.7)$ & $9.2(9.1-9.4)$ & $9.3(9.1-9.6)$ & 0.251 \\
\hline Phosphate, mg/dL $(N=20,24,44)$ & $4(3.5-4.5)$ & $3.4(3.1-4.1)$ & $3.6(3.2-4.3)$ & $0.016^{* *}$ \\
\hline Creatinine, mg/dL $(N=36,39,75)$ & $0.91(0.9-1)$ & $0.9(0.8-1)$ & $0.9(0.9-1)$ & 0.053 \\
\hline Urinary calcium excretion, mg/24 $\mathrm{h}(N=4,1,5)$ & 84.5 (22.6-98) & $123(123-123)$ & $89(41.7-112)$ & 0.400 \\
\hline Urinary phosphate excretion, $\mathrm{mg} / 24 \mathrm{~h}(\mathrm{~N}=3,0,3)$ & $35(32.6)$ & - & $35(32.6)$ & - \\
\hline
\end{tabular}

*GT positive genetic test, $-G T$ negative genetic test, ALP Alkaline phosphatase. ${ }^{*}$ Significant statistical differences between groups 
Table 5 Diagnostic utility measures for serum ALP level thresholds

\begin{tabular}{lllllll}
\hline ALPa levels & Sensitivity & Especificity & PPV & NVP & $+L R$ & $-L R$ \\
\hline$<20$ IU/L & $15.4 \%$ & $100 \%$ & $100 \%$ & $57.7 \%$ & Infinity & 0.85 \\
$<25$ IU $/ L$ & $43.6 \%$ & $97.8 \%$ & $94.4 \%$ & $66.7 \%$ & 19.8 & 0.58 \\
$<30$ IU/L & $82.1 \%$ & $44.4 \%$ & $56.1 \%$ & $74.1 \%$ & 1.48 & 0.4 \\
$<35 \mathrm{IU} / \mathrm{L}$ & $97.4 \%$ & $4.4 \%$ & $46.9 \%$ & $66.7 \%$ & 1.02 & 0.59 \\
\hline
\end{tabular}

PPV positive predictive value, NPV negative predictive value, $L R$ likelihood ratio, $A L P$ Alkaline phosphatase

identifying the presence of an $A L P L$ variant. In contrast, ALP levels seem to be discriminative enough for this purpose. ALP levels below $25 \mathrm{IU} / \mathrm{L}$ showed a high specificity (97.8\%), positive predictive value (94.4\%) and positive likelihood ratio (19.8). Consequently, in the presence of symptoms potentially related to HPP and biochemical abnormalities, specific ALP cut-off levels could help diagnostic strategies, especially when genetic testing is not available. If these results are confirmed in other populations, these key findings could prove very useful in clinical practice.

With regards to clinical features, only the presence of musculoskeletal pain was significantly and strongly associated with a positive genetic ALPL status. Previous publications also showed a high incidence (41-95\%) of musculoskeletal pain in subjects with HPP [5, 15-17]. Indeed, Shapiro et al. recently described musculoskeletal pain attributable to HPP requiring pain medications, such as opioids, as one of the scenarios necessitating treatment with enzyme replacement [21]. However, given the high prevalence of pain in the overall population with hypophosphataseamia, the etiology and characteristics of pain in HPP should be characterized before therapeutic intervention. Metatarsal stress fractures were also more frequent in individuals with $A L P L$ disease-causing variants. In a recent publication, metatarsal fractures reached a prevalence of $21 \%$ and were considered typical of HPP [16]. The number of subjects presenting this feature was limited and did not allow for any definitive conclusions. Nevertheless, it was the only distinctive clinical symptom related to a positive genetic result when ALP levels were above $25 \mathrm{IU} / \mathrm{L}$.

On the other hand, to implement strategies that will enable an early detection of the disease is mandatory. In our study, a significant 19-year delay was observed, which is in accordance with the global HPP Registry, the largest observational study including real-world data cases [15]. Our study aimed to highlight the distinct clinical characteristics of two groups of subjects with the same biochemical abnormality but with a different genetic status. Clarifying this aspect is an important issue from the diagnostic point of view, given the high clinical variability of the disease [22], the evidence of normal substrates in some adults carrying a defective $A L P L$ allele and the limitations of conventional genetic studies [18, 23].
The main weaknesses of our study are the lack of pediatric medical histories, the use of a clinical questionnaire for identifying clinical symptoms and limited data available regarding certain variables, such as radiographic records. As a limitation, the design of the study does not allow us to establish a definitive clinical diagnosis of HPP: although a detailed questionnaire was designed for the evaluation of subjects included, a complete visit and complementary tests targeted to study clinical features or laboratory abnormalities were not performed, so that some subjects with variants in $A L P L$ could be carriers. Furthermore, ALP substrates and the analysis of the dominant negative effect of the variants have not been performed due to limitations in their determination.

The main strengths are the thorough analysis of medical records, which yielded a considerably large sample size for a rare disease, the integration of data across a wide range of variables, the analysis of information obtained through a detailed clinical questionnaire and the genetic analysis encompassing this large population. In addition, to the best of our knowledge, this is the first time that ALP cut-off levels are used to identify a positive $A L P L$ genetic test, although these results need further investigation.

\section{Conclusions}

In subjects with persistent hypophosphatasaemia, one out of two individuals with primary low ALP levels, presented an $A L P L$ disease-causing variant. It is imperative that the clinical and biochemical characteristics and its predictive value are defined if early diagnoses are to be obtained and erroneous therapeutic decisions avoided. Musculoskeletal pain and ALP levels below or equal to $25 \mathrm{IU} / \mathrm{L}$ are associated with a positive result in $A L P L$ genetic testing. In this scenario, ALP levels below this threshold seem to be very useful for predicting the presence of $A L P L$ disease-causing variant. If confirmed in other populations, these key findings can be useful in clinical practice. Prospective studies evaluating these findings and establishing the natural evolution of HPP will definitely enable a comprehensive determination of the disease's complete clinical spectrum in adults.

\section{Methods}

\section{Study population and design}

This cross-sectional study was performed at La Paz University Hospital (Madrid, Spain). The study adhered to the tenets of the Declaration of Helsinki and approval was obtained from its ethics committee. Each subject provided written informed consent prior to inclusion.

First, 1,536,711 laboratory records including ALP serum levels values from 386,353 subjects were screened to identify abnormally low ALP results. These values were recorded in the biochemical database of the hospital from 
2009 to 2015. The main criteria for inclusion were: adults aged 18 years or older with persistent hypophosphatasaemia, defined as $\geq 2$ ALP measurements below or equal to $35 \mathrm{IU} / \mathrm{L}$. Subjects were excluded if $\geq 1$ ALP values were above $45 \mathrm{IU} / \mathrm{L}$ (lower limit for adults), or if clinical records revealed secondary underlying causes of hypophosphatasaemia, such as cancer or chemotherapy, adynamic renal osteodystrophy, severe hypothyroidism, Cushing's disease, Wilson's disease, nutritional deficiencies, hypomagnesemia, hypozincemia, myocardial infarction, sepsis, major trauma or surgery, massive transfusions and clofibrate therapy among others [11].

\section{Collected data}

Eligible subjects completed a questionnaire to report family or personal history of musculoskeletal pain, muscle weakness, fractures, premature dental loss and previous orthopedic surgery, among other clinical features classically associated with HPP. Additionally, radiographic data were retrieved from medical records. Musculoskeletal pain was considered when symptoms were recurring or chronic (>6 months) and not when transient and muscle weakness was defined by a chronic subjective perception of a decrease in muscle strength, but no physical examination was performed. Dental abnormalities were defined as tooth shape abnormalities, structure and colour abnormalities of enamel or dentin, thin enamel, late teeth eruption or severe/recurrent cavities and early loss of permanent teeth was defined as the loss of several teeth or extraction $(>10)$ due to tooth abnormalities in the past, prior to the age of 50 . In terms of fractures, peripheral traumatic and fragility ones were evaluated as were stress metatarsal and atypical fractures. Multiple fractures were defined as more than three. Chondrocalcinosis and calcific periarthritis were considered when the subject had a previous diagnosis or if a prior imaging test confirmed the diagnosis. Subjects were required to grade their pain on a $100 \mathrm{~mm}$ visual analog scale (VAS) and to complete the Health Assessment Questionnaire-Disability Index (HAQ-DI). Individuals were requested to provide a blood sample for the genetic analysis.

\section{Laboratory methods}

Between 2009 and 2013, the University La Paz Hospital Laboratory utilized an Olympus 5400 analyzer (Beckman Coulter) to measure serum ALP activity. In February 2014, it switched to Siemens Healthineers (Advia 2400 chemistry system) and clinically acceptable correlation and comparison was demonstrated between the two devices (internal Laboratory data available). Both methods measure ALP activity by a kinetic rate method in which pnitrophenyl phosphate (a colourless organic phosphate ester substrate) is hydrolyzed by ALP to the yellow- coloured product pnitrophenol and phosphate at $\mathrm{pH}$ 10,3. Enzymatic activity of ALP is directly proportional to changes in absorbance at $410 \mathrm{~nm}$. The normal adult's range is 45 to $116 \mathrm{IU} / \mathrm{L}$.

\section{Genetic analysis}

Genomic DNA was extracted from peripheral blood with a Chemagic Blood kit (Perkin Elmer, Waltham, MA) and the screening of the exons and intron/exon boundaries of ALPL (NM_000478.4) was performed by Sanger sequencing. In silico pathogenicity prediction and control population frequency analysis were assessed using Alamut V2.6 software (Interactive Biosoftware Rouen, France), Varsome (https://varsome.com/), CADD software (http:// cadd.gs.washington.edu) and Silvent et al. criteria [24]. The allelic frequencies were determined using gnomAD (http://gnomad.broadinstitute.org/) and the in silico tools included CADD V1.3, DANN, SIFT, Polyphen, MutationTester, Mutation assessor, FATHM and SpliceSiteFinderlike, MaxEntScan, NNSPLICE, GeneSplicer. The ALPL disease-causing variants database (http://www.se-sep.uvsq. fr/03_hypo_mutations.php) was also consulted to obtain up-to-date information about the genetic variants included in our study (Additional file 1: Table S1) already identified [25-36]. Variants were classified according to the American College of Medical Genetics and Genomics (ACMG) standards and guidelines [20].

\section{Statistical analysis}

First, descriptive analysis was employed to determine the prevalence of $A L P L$ variants among subjects with persistent hypophosphatasaemia and to compare clinical and laboratory characteristics between individuals with and without $A L P L$ variants. Continuous variables were described as median (interquartile range -IQR-) and categorical variables as an absolute number and relative percentage. Comparisons between two independent groups for continuous variables were performed using the Student's $t$-test for unpaired data if normally distributed, or a Mann-Whitney $U$ test when not. Statistical significance difference between groups for categorical variables was calculated using the Chi-square test or the Fisher's exact test, as appropriate. Logistic regression models adjusted for confounders were employed to investigate the association between clinical and laboratory characteristics and the genetic status. Finally, diagnostic utility measures (sensitivity, specificity, positive and negative predictive value and positive and negative likelihood ratio) to predict the likelihood of having an $A L P L$ variant were calculated. The level of statistical significance was set at $p<0.05$. Statistical analyses were performed using the IBM SPSS Statistics 23.0 for Windows. 


\section{Supplementary information}

Supplementary information accompanies this paper at https://doi.org/10. 1186/s13023-020-1315-y

Additional file 1: Table S1. List of subjects displaying ALPL disease causing variants observed in the cohort according to the transcript NM_000478.4, clinical features associated and family history available.

Additional file 2: Table S2. Diagnostic utility measures for each of the symptoms combined with ALP levels between 25 and $35 \mathrm{IU} / \mathrm{L}$.

\section{Acknowledgements}

Carla Granados from Trialance SCCL and the translation service of the Spanish Society of Rheumatology provided medical writing assistance.

\section{Authors' contributions}

PA conceived the idea for this study and participated in its design and coordination, supervising all the process. CT, PA, SG, IM, CP and AB recruited and evaluated the subjects. JT, KH and PL performed and interpreted the genetic tests. $A B$ and $J \mathrm{MI}$ contributed to the recruitment process and evaluated the biochemical results. CT, PA and VNC analyzed and interpreted the data and conceived, designed and drafted the manuscript. All authors were involved in revising the manuscript, gave final approval of the version to be published and agreed to be accountable for all aspects of their work.

\section{Funding}

Genetic testing was supported by a grant from Alexion Pharmaceuticals Inc., which had no role in the study design or data analysis.

\section{Availability of data and materials}

The datasets generated and/or analyzed during the current study are available from the corresponding author on reasonable request.

\section{Ethics approval and consent to participate}

The study adhered to the tenets of the Declaration of Helsinki and approval was obtained from La Paz University Hospital's ethics committee. Each subject provided written informed consent prior to inclusion.

\section{Consent for publication}

Not applicable

\section{Competing interests}

P. Aguado has received fees for advisory board participation from Alexion (Spain) and is a recipient of an unrestricted research grant from this pharmaceutical company, but not for this manuscript. The rest of authors declare they do not have any other conflict of interest regarding the publication of this article.

\section{Author details}

'Department of Rheumatology, La Paz University Hospital, IdiPAZ, Paseo de la Castellana, 261, 28046 Madrid, Spain. ${ }^{2}$ Institute of Medical and Molecular Genetics (INGEMM), La Paz University Hospital, IdiPAZ, Universidad Autónoma de Madrid, Madrid, Spain. ${ }^{3}$ CIBERER (Centro de Investigación Biomédica en Red de Enfermedades Raras), ISCIII, Madrid, Spain. ${ }^{4}$ Department of Clinical Biochemistry, La Paz University Hospital, Madrid, Spain. ${ }^{5}$ Skeleta dysplasia multidisciplinary Unit (UMDE), La Paz University Hospital, Madrid, Spain.

Received: 31 August 2019 Accepted: 24 January 2020

Published online: 17 February 2020

References

1. Whyte MP. Physiological role of alkaline phosphatase explored in hypophosphatasia. Ann N Y Acad Sci. 2010;1192:190-200.

2. Millán JL, Whyte MP. Alkaline phosphatase and Hypophosphatasia. Calcif Tissue Int. 2016:98(4):398-416.

3. Whyte MP. Hypophosphatasia: an overview for 2017. Bone. 2017 102(2016):15-25.

4. Mornet E, Yvard A, Taillandier A, Fauvert D, Simon-Bouy B. A molecularbased estimation of the prevalence of Hypophosphatasia in the European population. Ann Hum Genet. 2011;75(3):439-45.
5. Berkseth KE, Tebben PJ, Drake MT, Hefferan TE, Jewison DE, Wermers RA. Clinical spectrum of hypophosphatasia diagnosed in adults. Bone. 2013; 54(1):21-7.

6. Maman E, Borderie D, Roux C, Briot K. Absence of recognition of low alkaline phosphatase level in a tertiary care hospital. Osteoporos Int. 2016; 27(3):1251-4.

7. Guañabens N, Mumm S, Möller I, González-Roca E, Peris P, Demertzis JL, et al. Calcific Periarthritis as the only clinical manifestation of Hypophosphatasia in middle-aged sisters. J Bone Miner Res. 2014;29(4):929-34.

8. Mornet E. Genetics of Hypophosphatasia. Clin Rev Bone Miner Metab. 2013; 11(2):71-7.

9. The Tissue Nonspecific Alkaline Phosphatase Gene Mutations Database. http://www.sesep.uvsq.fr/03_hypo_mutations.php.

10. Hofmann C, Girschick H, Mornet E, Schneider D, Jakob F, Mentrup B. Unexpected high intrafamilial phenotypic variability observed in hypophosphatasia. Eur J Hum Genet. 2014;22(10):1160-4.

11. McKiernan FE, Berg RL, Fuehrer J. Clinical and radiographic findings in adults with persistent hypophosphatasaemia. J Bone Miner Res. 2014 Jul:29(7):1651-60.

12. Sutton RAL, Mumm S, Coburn SP, Ericson KL, Whyte MP. "Atypical femoral fractures" during bisphosphonate exposure in adult hypophosphatasia. J Bone Miner Res. 2012;27(5):987-94.

13. Cundy T, Michigami T, Tachikawa K, Dray M, Collins JF, Paschalis EP, et al. Reversible deterioration in Hypophosphatasia caused by renal failure with bisphosphonate treatment. J Bone Miner Res. 2015;30(9):1726-37.

14. Mori M, DeArmey SL, Weber TJ, Kishnani PS. Case series: Odontohypophosphatasia or missed diagnosis of childhood/adult-onset hypophosphatasia? - Call for a long-term follow-up of premature loss of primary teeth. Bone Rep. 2016;5:228-32.

15. Högler W, Langman C, Gomes Da Silva H, Fang S, Linglart A, et al. Diagnostic delay is common among patients with hypophosphatasia: initial findings from a longitudinal, prospective, global registry. BMC Musculoskelet Disord. 2019;20(1):1-9.

16. Schmidt T, Mussawy H, Rolvien T, Hawellek T, Hubert J, Rüther W, et al. Clinical, radiographic and biochemical characteristics of adult hypophosphatasia. Osteoporos Int. 2017:28(9):2653-62.

17. Weber TJ, Sawyer EK, Moseley S, Odrljin T, Kishnani PS. Burden of disease in adult patients with hypophosphatasia: results from two patient-reported surveys. Metabolism. 2016;65(10):1522-30.

18. Riancho-Zarrabeitia L, García-Unzueta M, Tenorio JA, Gómez-Gerique JA, Ruiz Pérez VL, Heath KE, et al. Clinical, biochemical and geneticspectrum of low alkaline phosphatase levels in adults. Eur J Intern Med. 2016:29:40-5.

19. McKiernan FE, Dong J, Berg RL, Scotty E, Mundt P, Larson L, et al. Mutational and biochemical findings in adults with persistent hypophosphatasaemia. Osteoporos Int. 2017:28(8):2343-8.

20. Richards S, Aziz N, Bale S, et al. Standards and guidelines for the interpretation of sequence variants: a joint consensus recommendation of the American College of Medical Genetics and Genomics and the Association for Molecular Pathology. Genet Med. 2015;17:405-24. https:// doi.org/10.1038/gim.2015.30

21. Shapiro JR, Lewiecki EM. Hypophosphatasia in adults: clinical assessment and treatment considerations. J Bone Miner Res. 2017;32(10):1977-80.

22. Whyte MP. Hypophosphatasia - aetiology, nosology, pathogenesis, diagnosis and treatment. Nat Rev Endocrinol. 2016;12(4):233-46.

23. Taillandier A, Domingues C, De Cazanove C, Porquet-Bordes V, Monnot S, Kiffer-Moreira T, et al. Molecular diagnosis of hypophosphatasia and differential diagnosis by targeted next generation sequencing. Mol Genet Metab. 2015;116(3):215-20.

24. Silvent J, Gasse B, Mornet E, Sire J-Y. Molecular evolution of the tissuenonspecific alkaline phosphatase allows prediction and validation of missense mutations responsible for Hypophosphatasia. J Biol Chem. 2014; 289(35):24168-79.

25. Mornet E, Taillandier A, Peyramaure S, Kaper F, Muller F, Brenner R, et al. Identification of fifteen novel mutations in the tissue-nonspecific alkaline phosphatase (TNSALP) gene in European patients with severe hypophosphatasia. Eur J Hum Genet. 1998;6(4):308-14.

26. Whyte MP, Zhang F, Wenkert D, McAlister WH, Mack KE, Benigno MC, et al. Hypophosphatasia: validation and expansion of the clinical nosology for children from 25years experience with 173 pediatric patients. Bone. 2015;75:229-39.

27. Ozono K, Yamagata M, Michigami T, Nakajima S, Sakai N, Cai G, et al. Identification of novel missense mutations (Phe310Leu and 
Gly439Arg) in a neonatal case of hypophosphatasia. J Clin Endocrinol Metab. 1996;81(12):4458-61.

28. Mumm S, Jones J, Finnegan P, Henthorn PS, Podgornik MN, Whyte MP. Denaturing gradient gel electrophoresis analysis of the tissue nonspecific alkaline phosphatase isoenzyme gene in hypophosphatasia. Mol Genet Metab. 2002;75(2):143-53.

29. Tenorio J, Álvarez I, Riancho-Zarrabeitia L, Martos-Moreno G, Mandrile G, de la Flor CM, et al. Molecular and clinical analysis of ALPL in a cohort of patients with suspicion of Hypophosphatasia. Am J Med Genet Part A. 2017;173(3):601-10.

30. Taillandier A, Lia-Baldini AS, Mouchard M, Robin B, Muller F, Simon-Bouy B, et al. Twelve novel mutations in the tissue-nonspecific alkaline phosphatase gene (ALPL) in patients with various forms of hypophosphatasia. Hum Mutat. 2001;18(1):83-4.

31. Orimo H, Hayashi Z, Watanabe A, Hirayama T, Hirayama T. Novel missense and frameshift mutations in the tissue-nonspecific alkaline phosphatase gene in a Japanese patient with hypophosphatasia. Hum Mol Genet. 1994;3:1683-4.

32. Spentchian M, Merrien $Y$, Herasse M, Dobbie Z, Gläser D, Holder SE, et al. Severe hypophosphatasia: characterization of fifteen novel mutations in the ALPL gene. Hum Mutat. 2003;22(1):105-6.

33. Orimo H, Goseki-Sone M, Sato S, Shimada T. Detection of deletion 1154 1156 hypophosphatasia mutation using TNSALP exon amplification. Genomics. 1997;42(2):364-6.

34. Taillandier A, Cozien E, Muller F, Merrien Y, Bonnin E, Fribourg C, et al. Fifteen new mutations (-195C\&gt;T, L-12X, 298-2A\&gt;G, T117N, A159T, R229S, 997+2T\&gt;A, E274X, A331T, H364R, D389G, 1256delC, R433H, N461I, C472S) in the tissue-nonspecific alkaline phosphatase (TNSALP) gene in patients with hypophosphatasia. Hum Mutat. 2000;15(3):293.

35. Henthorn PS, Raducha M, Fedde KN, Lafferty MA, Whyte MP. Different missense mutations at the tissue-nonspecific alkaline phosphatase gene locus in autosomal recessively inherited forms of mild and severe hypophosphatasia. Proc Natl Acad Sci U S A. 1992;89(20):9924-8.

36. Taillandier A, Zurutuza L, Muller F, Simon-Bouy B, Serre JL, Bird L, et al. Characterization of eleven novel mutations (M45L, R119H, 544delG, G145V, H154Y, C184Y, D289V, 862+5A, 1172delC, R411X, E459K) in the tissuenonspecific alkaline phosphatase (TNSALP) gene in patients with severe hypophosphatasia. Hum Mutat. 1999;13(2):171-2.

\section{Publisher's Note}

Springer Nature remains neutral with regard to jurisdictional claims in published maps and institutional affiliations.

Ready to submit your research? Choose BMC and benefit from:

- fast, convenient online submission

- thorough peer review by experienced researchers in your field

- rapid publication on acceptance

- support for research data, including large and complex data types

- gold Open Access which fosters wider collaboration and increased citations

- maximum visibility for your research: over $100 \mathrm{M}$ website views per year

At BMC, research is always in progress.

Learn more biomedcentral.com/submissions 